\title{
Properties of magnetic nanoparticles in vehicle-derived aerosol
}

\author{
ILONA NYIRO-KOSA, MIHÁLY PÓSFAI AND FAIZAN \\ AHMAD
}

University of Pannonia

Presenting Author: kosaili@gmail.com

Properties of magnetic nanoparticles in vehicle-derived aerosol

Nyirő-Kósa, I. ${ }^{1}$, Ahmad, F., Pósfai, M. ${ }^{1}$

1 Research Institute of Biomolecular and Chemical Engineering, University of Pannonia, Veszprém, Hungary; kosaili@gmail.com, faizan_afd@yahoo.com, mihaly.posfai@gmail.com

Magnetic particles constitute a minor but important fraction of the atmospheric aerosol. By studying magnetic proxies of windblown dust, climate changes over geologic timescales can be studied; on the other hand, anthropogenic magnetic particles can serve as tracers of particulate pollution. Concern about potential health effects of airborne magnetic particles was raised by their identification in the human brain, presumably originating from atmospheric pollution [1]. The goal of this study was to characterize the morphologies, sizes, compositions and spatial associations of vehicle-derived magnetic particles. We used a simple device to collect the magnetic particles separately from other constituents of the aerosol, preserving their original states. Particle properties were studied using electron microscopy techniques (SEM, TEM, STEM).

Particles were collected (1) from the urban atmosphere, (2) inside a car while driving on a highway, (3) from exhaust emission, and (4) from the brake wear. The particles from the urban atmosphere appear to be identical to the ones found in tailpipe emissions. In both types of samples the magnetic particles are 1-4 $\mu \mathrm{m}$ aggregates composed of iron oxide nanocrystals (7-11 nm, mainly magnetite) that are typically wrapped in layers of carbonaceous material. Magnetic fraction of the brake wear differs from tailpipe particles in terms of sizes, minor element compositions and mineral phase, and even the degree of ordering of carbonaceous material. Brake wear particles seem to have originated primarily from the corrosion of the brake disc rather than from the brake pad. The characteristics of the iron oxides found in the car cabin sample are very similar to those in the exhaust and brake wear samples. Although single magnetite nanoparticles were not detected in the samples, the primary magnetic agglomerates are still small enough to reach the lungs upon inhalation, potentially causing various deteriorative health effects.

[1] Maher, B.A., Ahmed, I.A.M., Karloukovski, V., MacLaren, D.A., Foulds, P.G., Allsop, D., Mann, D.M.A., Torres-Jardón, R., Calderon-Garciduenas, L. (2016) Proc. Natl. Acad. Sci. U.S.A., 113, 10797-10801. 